\title{
Osler's node on the thigh, an uncommon location, but a valuable diagnostic aid
}

\author{
Benjamin Davido $\cdot$ Nicolas Davido • \\ Anne-Claude Cremieux • Pierre de Truchis • \\ Christian Perronne
}

Received: 30 January 2014/ Accepted: 17 February 2014/Published online: 26 February 2014

(C) SIMI 2014

A 65-year-old man without any significant past medical history, presented with fever, fatigue and weight loss. He complained of a painful macular violaceous lesion of $2 \mathrm{~cm}$ (Fig. 1) located on the left thigh, negative to compression. The remaining physical examination was unremarkable except for a slight systolic mitral murmur and conjunctival hemorrhages.

Considering the cutaneous signs although atypical, could be integrated in the Duke minor criteria, we suspected a possible endocarditis. Hence, after blood cultures, we started antibiotics using IV amoxicillin $(200 \mathrm{mg} / \mathrm{kg} /$ day) and gentamycin while scheduling echocardiography. Subsequent laboratory tests confirmed two blood cultures positive for Streptococcus gordonii.

Transesophageal echocardiography showed 2 mobile vegetations of 8 and $17 \mathrm{~mm}$ on the mitral valve, which justified an urgent mitral valve surgery within the next $24 \mathrm{~h}$.

During the first week after treatment, we observed a fast disappearance of the cutaneous lesion.

The patient recovered after 4 weeks of medical care, followed by removal of a dental granuloma, and is considered healed at 3 years' follow-up.

Is it an Osler's node or a Janeway lesion? Sir William Osler, in 1893, described the painful sign with his name

B. Davido $(\square)$ - A.-C. Cremieux · P. de Truchis · C. Perronne Infectious Diseases Department, Raymond Poincaré Teaching Hospital, 92380 Garches, France

e-mail: benjamin.davido@gmail.com;

benjamin.davido@rpc.aphp.fr

N. Davido

Odontology Department, Pitié Salpétrière Teaching Hospital, Paris, France associated ever since. Osler's nodes are painful and slightly raised erythematous nodules, from $1 \mathrm{~mm}$ to more than $1 \mathrm{~cm}$ [1], commonly located on the pads of the fingers and toes. Sometimes they are located on the skin of the forearms, flank and trunk [2].

Then in 1899, Janeway described a different non-tender lesion found on the palms and soles. The macules, usually hemorrhagic, are non-tender, have a variable size, and remain longer than Osler's nodes [3].

Osler's node on the thigh has never been reported before. Despite some atypical findings, various characteristics of the skin lesion including its size, its shape and its marked painful character led us to conclude this was an Osler's node.

Moreover, the histologic findings do not always allow the physician to distinguish between an Osler's node and a Janeway lesion. Several theories have been hypothesized for the formation of the cutaneous lesions, and therefore, there is no consensus for specific histologic signs. Nowadays septic or bland microemboli seem to explain Osler's nodes [4] leading sometimes to dermal microabscesses with possible isolation of the pathogenic organism from aspirates [5]. However, a phenomenon of an immunologically complex vasculitis is possibility.

Osler's nodes and Janeway lesions might even be the result of the same process leading to different lesions according to the micro-anatomic site, and the delay in proceeding to biopsy. The virulence of the microorganism responsible for an acute or subacute infective endocarditis may also play a role in the type of histologic manifestations.

In conclusion, our report suggests that a meticulous physical examination may allow finding these legendary cutaneous signs that are helpful clues to lead quickly to 


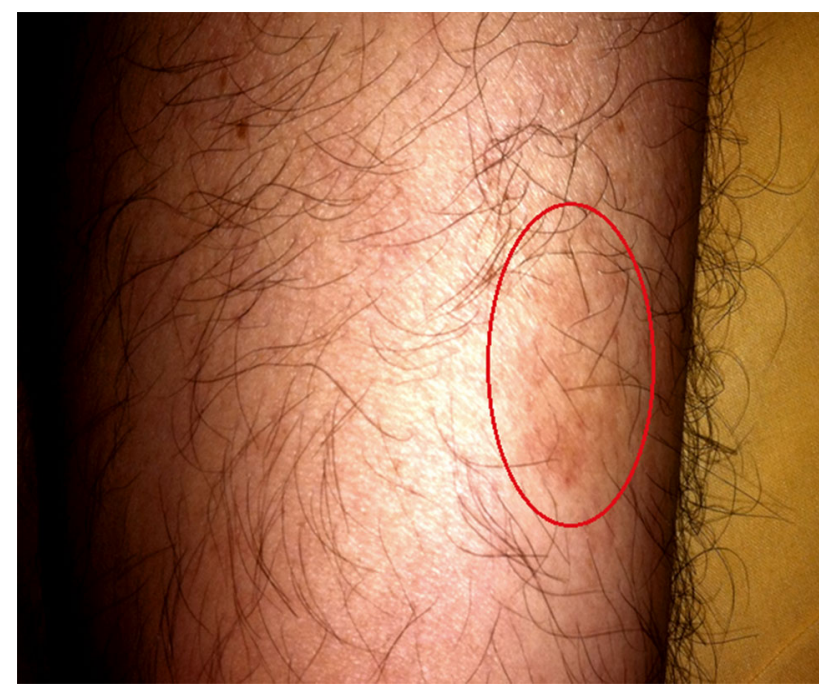

Fig. 1 Violaceous macular painful lesion on the left thigh representing an Osler's node

the diagnosis of infectious endocarditis, or to isolate the bacteria in difficult cases by a simple biopsy of the lesion [5].

\section{Conflict of interest None.}

Ethical standard This manuscript also fulfills the ethics committee approval.

\section{References}

1. Howard EJ (1960) Osler's nodes. Am Heart J 59:633-634

2. Urbano FL (2000) Peripheral signs of endocarditis. Hosp Physician 36:41-46

3. Gil MP, Velasco M, Botella R, Ballester JE, Pedro F, Aliaga A (1993) Janeway lesions: differential diagnosis with Osler's nodes. Int J Dermatol 32:673-674

4. Yee J, McAllister CK (1987) Osler's nodes and the recognition of infective endocarditis: a lesion of diagnostic importance. South Med J 80:753-757

5. Alpert JS, Krous HF, Dalen JE, O'Rouke RA, Bloor CM (1976) Pathogenesis of Osler's nodes. Ann Intern Med 85:471-473 